\title{
Minimally Invasive Surgical Treatment for Severe Symptomatic Lumbar Spinal Stenosis: A Case Study \\ Michael W Perry ${ }^{1}$, Elizabeth M Hudak ${ }^{2 *}$ and Timothy A Luke
}

${ }^{1}$ Medical Director, Laser Spine Institute LLC, Tampa, Florida, USA

${ }^{2}$ Medical Research Assistant, Laser Spine Institute LLC, Tampa, Florida, USA

${ }^{3}$ Orthopedic Spine Surgeon, Laser Spine Institute LLC, Tampa, Florida, USA

\begin{abstract}
Background: Minimally invasive spine surgeries using endoscopic techniques have shown to be effective at treating lumbar spinal stenosis. However, there lacks evidence that bilateral decompression of the nerve root can be achieved through a unilateral endoscopic technique. Thus, this case study examines whether an outpatient surgical treatment for severe lumbar spinal stenosis (LSS) requiring bilateral decompression through a unilateral approach can be performed endoscopically.

Methods: A 63-year old non-smoking African American male presented with symptoms of pain in the left buttock that radiated into the posterior left thigh. Magnetic resonance imaging (MRI) confirmed severe L4/5 spinal stenosis bilaterally. The patient underwent out-patient minimally invasive unilateral laminotomy for bilateral L4/5 decompression of central canal stenosis. This procedure included a partial facetectomy with removal of the contralateral ligamentum flavum, and decompression of the lateral recesses.

Results: The procedure lasted one hour and 16 minutes. Post-operative MRI confirmed bilateral decompression of the spinal canal. The patient tolerated the surgery well and was released two hours post-operative awake and in stable condition. There were no operative complications and an estimated blood loss of 25 millilitres. The patient reported the ability to walk with complete resolution of ridiculer pain, tingling and numbness the same day as surgery as well as at 3-, 6- and 18-months post-operatively.

Conclusion: This case study indicates that an outpatient endoscopic unilateral laminotomy for bilateral decompression of the central canal and lateral recesses is effective at reducing pain and disability level immediately following surgery and up to 18-months post-operative. Results also indicate that this outpatient procedure can treat severe LSS with short operative times, no operative complications, and minimal blood loss.
\end{abstract}

Keywords: Decompression; Endoscopy; Laminotomy; Foraminotomy; Back pain; Bilateral decompression; Nerve root; Spinal canal

\section{Introduction}

First described by Baily and Casamajor in 1911 [1], lumbar spinal stenosis (LSS) is the narrowing of the spinal canal caused by age-related degenerative processes such as bony overgrowth, enlargement of the facet joints, ligamentum flavum hypertrophy, or bulging and herniated discs [2-6]. As degenerative processes cause the spinal canal to narrow, the neural elements within the canal can get compressed. This typically results in intense back pain, neurogenic claudication, and radicular symptoms [7].

Symptomatic LSS can be treated with a variety of different modalities. Conservative treatment can consist of physical therapy, pain management, chiropractic care, acupuncture, and medications. When conservative treatments fail, surgery would then be an option. The Spine Patient Outcomes Research Trial (SPORT) [8,9] compared conservative treatment of LSS with a surgical intervention. Follow-up analyses at both 2 - and 4-years post-operative indicated that patients that underwent surgical correction of LSS fared better than those who received conventional treatments.

Although invasive open surgery (i.e., laminectomy) is considered the conventional treatment for LSS, out-patient minimally invasive spine surgery (MIS) has been evolving over the past few decades. Studies indicate that MIS for the treatment of LSS is as effective at providing satisfactory decompression as open surgery without adverse effects including damage to the posterior ligamentum, muscles and tissues, dural leaks, and large incisions that are associated with open surgery [10-17]. This is important because the weaknesses caused by the extensive surgical dissection and muscle detachment have sometimes lead to paraspinal muscle denervation and atrophy; which is correlated with an increased incidence of "failed back syndrome" and chronic pain $[18,19]$.

Standard out-patient MIS using endoscopy for the treatment of LSS does not require a large degree of bone or ligament removal. However, in more severe cases in which bilateral decompression is needed, more bone removal is required to obtain sufficient decompression. The use of an endoscopic procedure to achieve bilateral decompression has been examined in a study by Çelik [20]. In this study, patients diagnosed with severe LSS were randomized to undergo a total laminectomy (TL) or MIS using endoscopy to perform a bilateral laminotomy. After surgery, all patients were ambulatory the first day after surgery and post-operative imaging demonstrated adequate decompressions in both treatment conditions. Perioperative complications, post-operative instability, and the overall rate of dural injuries were all significantly higher among the TL group than the MIS condition $(p<.05)$. Although the study indicates that endoscopy, instead of open surgery, can be used

*Corresponding author: Elizabeth $\mathrm{M}$ Hudak, $\mathrm{PhD}$, Medical Research Assistant, Laser Spine Institute LLC, 3031 North Rocky Point Drive Eas Tampa, Florida 33607,USA, Tel: +813-289-9613 ext. 215; Fax: +813-4184237; E-mail: ehudak@laserspineinstitute.com

Received December 19, 2012; Accepted January 23, 2013; Published January 25, 2013

Citation: Perry MW, Hudak EM, Luke TA (2013) Minimally Invasive Surgical Treatment for Severe Symptomatic Lumbar Spinal Stenosis: A Case Study. J Spine 2: 130. doi:10.4172/2165-7939.1000130

Copyright: $\odot 2013$ Perry MW, et al. This is an open-access article distributed unde the terms of the Creative Commons Attribution License, which permits unrestricted use, distribution, and reproduction in any medium, provided the original author and source are credited. 
to achieve bilateral decompression through a bilateral laminotomy, the ability to achieve bilateral decompression through an outpatient unilateral laminotomy endoscopically is still uncertain.

In patients with severe LSS, the ability to provide complete bilateral decompression through a unilateral endoscopic approach has structural benefits including the preservation of the contralateral structures, lamina, and facet joint at the index surgery level [10]. To the best of our knowledge, Hong et al. [10] is the only study to compare unilateral and bilateral laminotomies for bilateral decompression in patients with LSS over 3-year post-operative. Results indicated that both unilateral and bilateral laminotomies provided adequate decompression and pain reduction. However, unilateral laminotomy was performed with shorter operative times, less blood loss, and induced less translational motion increase after surgery than patients who received the bilateral laminotomy. Thus, unilateral laminotomy, compared to bilateral laminotomy for bilateral decompression, may reduce the risk of late instability, result in less operative blood loss, and have a shorter operative time. However, this study did not examine whether a unilateral laminotomy can be performed as an outpatient procedure with only intravenous (IV) sedation, or whether it can reduce the level of disability experienced by patients.

This case study examines the use of an outpatient minimally invasive endoscopic procedure that utilizes a unilateral laminotomy to perform bilateral decompression of central canal stenosis with decompression of bilateral lateral recess for the treatment of severe symptomatic LSS.

\section{Methods}

Informed consent was obtained from the patient. Pre-operative and post-operative MRI (without contrast) were conducted. The scans were reviewed for evidence of LSS by independent radiologist blinded to the clinical results and unaffiliated with the operating institution.

\section{Outcome measures}

The Visual Analog Scale (VAS) [21] was used to measure pain intensity pre- and postoperatively. The Oswestry Disability Index (ODI) [22] was used to measure disability level pre- and postoperatively. To measure the safety of this surgical procedure, data pertaining to estimated blood loss (EBL), perioperative complications, and length of surgery were retrieved from the patients' medical records.

\section{Case report}

A 63-year old non-smoking African American male with a body mass index of 24.2 presented with symptoms of pain in the left buttock that radiated into the posterior left thigh. The patient denied that the pain was initiated from any injury or trauma but rather a gradual onset of symptoms that began approximately 1-year prior. Pain severity was reported to increase with activities such as walking or standing and was alleviated when assuming a seated or lying down position. As a practicing surgeon, the patient was finding it difficult to continue his practice due to the pain experienced while standing. Previous attempts to alleviate pain included the use of over-the-counter pain medications for six weeks with no significant pain relief. Other types of treatments such as physical therapy, chiropractic care, or acupuncture were not used by the patient as a treatment for pain. The patient did not have any prior surgical consults or surgeries pertaining to the spine.

Pre-operative MRI scans revealed severe central stenosis at the L4/5 level caused from both a congenital basis as well as the result of a bulging disk, bilateral facet, and ligamentum flavum hypertrophy. There was moderate biforaminal narrowing due to a disk bulge and osteophytic spurring. The L4/5 level had moderate to severe bilateral lateral recess stenosis which can be seen in figure 1 . Degenerative disc disease was noted at L2/3 and L5-S1. The MRI scans also revealed a bulging disk and foraminal narrowing at L2-L4 due to osteophytic spurring and a bulging disc. Imaging provided no evidence of malalignment or spondylolisthesis. Based off these findings, the patient was diagnosed with lumbar osteoarthritis, degenerative disc disease, and LSS. No other pertinent abnormalities were observed. Pre-operative MRI scans are presented in figure 1 .

After the physician informed the patient of the diagnosis, the physician explained available treatment options including conservative treatments (e.g., physical therapy), intensive open surgical procedures, and MIS using endoscopy to the patient in length. With MIS being the recommended treatment, it was explained in detail to the patient along with the potential risks and outcomes associated with it. The physician also stressed the importance of physical therapy as an adjunct to the procedure. After discussing all treatment options, the patient and physician agreed on pursuing the MIS procedure based on imaging and patient symptoms.

\section{Surgical procedure}

The patient underwent an outpatient MIS endoscopic left approach L4/5 bilateral laminotomy decompression of the central canal and bilateral lateral recess with left foraminotomy including partial facetectomy with the removal of the ipsilateral and majority contralateral ligamentum flavum. The patient was given IV sedation for the procedure.

The patient was brought to the surgical suite and placed in a prone position on the operating room table. The safety strap and monitors were applied. The patient's lumbar spine was then prepped with Chloraprep and draped in the usual sterile fashion. The C-arm was also draped. A time out was performed; the patient, procedure, level and approach were again verified by the surgeon and operating room team. A needle holder was used to mark the position of the decompression site at L4/5. The superficial skin was anesthetized with $1 \%$ lidocaine with epinephrine as well as penetrating deeper for the decompression site. An incision was made utilizing a \#15 blade; a $3 \mathrm{~cm}$ horizontal incision at the L4/5 level. The guide pin was placed at the left L4 lamina followed by the first dilating tube. This placement was verified by C-arm fluoroscopy in the anteroposterior (AP) view. Sequential dilating tubes were incorporated until the appropriate working tube was placed and remaining tubes were removed. The working tube
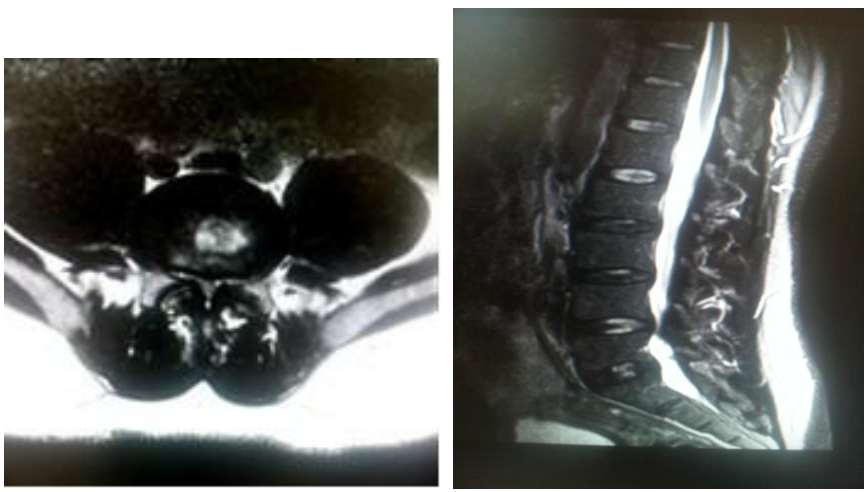

Figure 1: Pre-operative axial and sagittal magnetic resonance imaging showing severe lumbar spinal stenosis at the L4-5 level. 
and guide pin placement were documented with AP and lateral intraoperative fluoroscopy

The endoscope was placed for visualization. Soft tissue was removed using a combination of electrocautery, laser, and Ferris Smith straight biting rongeurs to clean the remaining soft tissue and expose the L4 lamina. The medial aspect of the facet joints and lamina were cleared of soft tissue in order to identify the superior lamina, medial facets and spinous process. Using a diamond tip burr, the least amount of bone necessary was removed from the superior lamina left, then undercutting the spinous process to gain access the right lamina, and the medial facet left. First the outer cortex was carefully burred followed by medullary bone. Then the inner cortex was identified and once confirmed, minimal burring was performed. When enough bone had been removed, the Kerrison rongeurs were first used between the ligamentum flavum, which was excessively hypertrophied on the left and right, and the superior lamina. The left and right superior laminar bones were partially removed and the ligamentum flavum was removed on the left and the majority on the right.

The Murphy probe was also used to probe the left and right traversing nerves in the lateral recess at the L4/5 level, which were narrowed by excessive osteophytes. Decompression of the traversing nerve roots with the burr and Kerrison rongeurs was carefully performed. Soft tissue and bone was removed until an adequate decompression of the lateral recess allowed the Murphy probe to easily follow the traversing nerves around the pedicles. We were also able to retract the dura medially to visualize the mobility of the traversing nerves on the left and the right.

When the decompression looked adequate by thorough inspection using the Murphy probe to verify central decompression along with bilateral lateral recess, the procedure was deemed complete. The area was thoroughly irrigated with antibiotic solution and aspirated throughout. Therapeutic steroid injection was performed. Marcaine $0.25 \%$ with epinephrine was injected into the skin and subcutaneous tissue around the incisions to aid in postoperative pain.

Following removal of the instrumentation, the incision and skin were closed with 2-0 antibacterial Vicryl, 3-0 Monocryl sutures and Steri-strips. Sterile $4 \times 4$ 's and Medipore tape was applied. All counts were correct. The patient was transferred to a stretcher, rolled to a supine position, and then escorted to the recovery room in stable condition.

\section{Results}

The procedure took one hour and 16 minutes to complete. The patient tolerated the surgery well and was released two hours postoperative awake and in stable condition. There were no $(0.00 \%)$ operative complications and an EBL of 25 millilitres was reported. Postoperative MRI (without contrast) of L4/5 confirmed decompression of the spinal canal (Figure 2). The patient reported the ability to walk with complete resolution of radicular pain, tingling and numbness the same day as surgery. Analysis indicate that scores on the VAS and ODI were significantly better post-operatively ( 0.00 and 0.00 respectively) than pre-operatively ( 3.25 and 17.77 respectively). In fact, the complete resolution of pain and disability ( 0.00 and 0.00 respectively) was reported at 3-, 6-, and 18-months post-operatively by the patient (Table 1).

\section{Discussion}

This case study examined the use of MIS for a patient with severe symptomatic LSS at L4/5 requiring decompression bilaterally

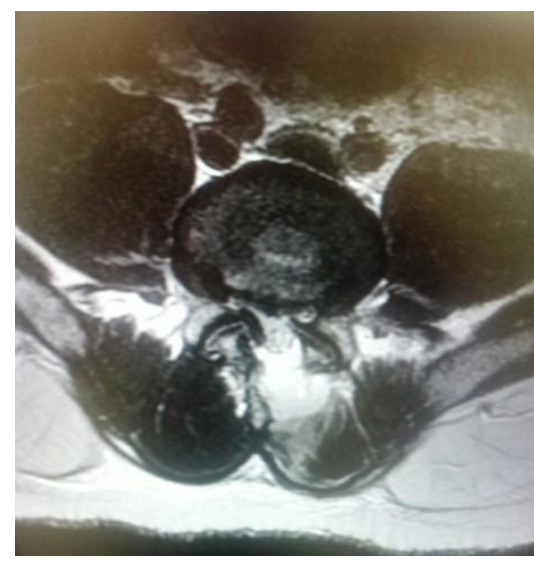

Figure 2: Post-operative axial magnetic resonance imaging showing complete decompression of lumbar spinal stenosis at the L4-5 level.

\begin{tabular}{|l|l|l|l|l|}
\hline \multirow{2}{*}{ Measures } & \multirow{2}{*}{ Pre-Operative } & \multicolumn{3}{|c|}{ Post-Operative } \\
\cline { 3 - 5 } & & 3-Months & 6-Months & 18-Months \\
\hline VAS $^{*}$ & 3.25 & 0.00 & 0.00 & 0.00 \\
\hline ODI $^{*}$ & 17.77 & 0.00 & 0.00 & 0.00 \\
\hline
\end{tabular}

*Lower scores indicate better pain and level of disability

Table 1: VAS and ODI Scores for a patient who underwent endoscopic minimally invasive surgery for severe symptomatic lumbar spinal stenosis.

performed unilaterally. The procedure resulted in a small incision, minimal soft-tissue injury, no operative complications, minimal blood loss, and the preservation of the posterior ligamentum and muscle. In addition to positive operative outcomes, this study provides evidence that MIS performed unilaterally for bilateral decompression is effective at resolving symptoms of pain. Even though the patient indicated a low level of pain on the VAS pre-operative (score of 3.25), he emphasized having a low pain tolerance and stated that his pain was sufficient enough to disrupt his ability to perform his occupational practice as a surgeon. The patient reported the absence of pain and disability immediately post-operative and at 3-, 6-, and 18-months post-operative.

The results of this study coincide with the findings from Hong et al. [10] that a unilateral laminotomy for bilateral decompression can result in short operative times, low operative blood loss, and a reduced level of pain. However, this study also indicates that this may also significantly reduce level of disability, and can be performed as an outpatient procedure using IV sedation. Successful decompression can be determined by either clinical outcomes or anatomical decompression as observed by post-operative imaging studies. This case study showed total decompression via MRI and total resolution of symptoms as indicated by his VAS and ODI scales. Confirmation of decompression from both MRI scans and patient self-report is important being that studies indicate that there is a poor association between imaging findings and the severity of clinical symptoms reported by patients [23]. According to patient feedback and post-operative MRI scans, this procedure was effective at achieving complete decompression.

\section{Conclusion}

This case study demonstrates the efficacy of performing an outpatient minimally invasive endoscopic bilateral laminotomy for the treatment of severe LSS. The small incision, minimal tissue injury, no operative complications or hospital stay, minimal blood loss, and preservation of the posterior ligamentum and muscle make this 
Citation: Perry MW, Hudak EM, Luke TA (2013) Minimally Invasive Surgical Treatment for Severe Symptomatic Lumbar Spinal Stenosis: A Case Study. J Spine 2: 130. doi:10.4172/2165-7939.1000130

Page 4 of 4

procedure a potentially safe surgical treatment for LSS. Although this is a single case study, the efficacy of the minimally invasive surgery for the treatment of LSS validates the need for future research with a larger sample size.

\section{References}

1. Bailey $P$, Casamajor $L$ (1911) Osteo-arthritis of the spine as a cause of compression of the spinal cord and its roots: with report of five cases. Journal of Nervous and Mental Disease 38: 588-609.

2. Epstein NE, Maldonado VC, Cusick JF (1998) Symptomatic lumbar spina stenosis. Surg Neurol 50: 3-10.

3. Alvarez JA, Hardy RH Jr (1998) Lumbar spine stenosis: a common cause of back and leg pain. Am Fam Physician 57: 1825-1834, 1839-40.

4. Postacchini $F$ (1985) The diagnosis of lumbar stenosis. Analysis of clinical and radiographic findings in 43 cases. Ital J Orthop Traumatol 11: 5-21.

5. Tan SB (2003) Spinal canal stenosis. Singapore Med J 44: 168-169.

6. Jacobsen S, Sonne-Holm S, Rovsing H, Monrad H, Gebuhr P (2007) Degenerative lumbar spondylolisthesis: an epidemiological perspective: the Copenhagen Osteoarthritis Study. Spine 32: 120-125.

7. Backstrom KM, Whitman JM, Flynn TW (2011) Lumbar spinal stenosisdiagnosis and management of the aging spine. Man Ther 16: 308-317.

8. Weinstein JN, Lurie JD, Tosteson TD, Tosteson AN, Blood EA, et al. (2008) Surgical versus nonoperative treatment for lumbar disc herniation: four-year results for the Spine Patient Outcomes Research Trial (SPORT). Spine (Phila Pa 1976) 33: 2789-2800

9. Weinstein JN, Tosteson TD, Lurie JD, Tosteson A, Blood E, et al. (2010) Surgical versus nonoperative treatment for lumbar spinal stenosis four-year results of the Spine Patient Outcomes Research Trial. Spine (Phila Pa 1976) 35: $1329-1338$

10. Hong SW, Choi KY, Ahn Y, Baek OK, Wang JC, et al. (2011) A comparison of unilateral and bilateral laminotomies for decompression of L4-L5 spinal stenosis. Spine (Phila Pa 1976) 36: E172-178.
11. Morgalla MH, Noak N, Merkle M, Tatagiba MS (2011) Lumbar spinal stenosis in elderly patients: is a unilateral microsurgical approach sufficient for decompression? J Neurosurg Spine 14: 305-312.

12. Yagi M, Okada E, Ninomiya K, Kihara M (2009) Postoperative outcome after modified unilateral-approach microendoscopic midline decompression for degenerative spinal stenosis. J Neurosurg Spine 10: 293-299.

13. Rosen DS, O'Toole JE, Eichholz KM, Hrubes M, Huo D, et al. (2007) Minimally invasive lumbar spinal decompression in the elderly: outcomes of 50 patients aged 75 years and older. Neurosurgery 60: 503-509.

14. Komp M, Hahn P, Merk H, Godolias G, Ruetten S (2011) Bilateral operation of lumbar degenerative central spinal stenosis in full-endoscopic interlaminar technique with unilateral approach: prospective 2-year results of 74 patients. $J$ Spinal Disord Tech 24: 281-287.

15. Oertel MF, Ryang YM, Korinth MC, Gilsbach JM, Rohde V (2006) Long-term results of microsurgical treatment of lumbar spinal stenosis by unilateral laminotomy for bilateral decompression. Neurosurgery 59: 1264-1269.

16. O'Toole JE, Eichholz KM, Fessler RG (2009) Surgical site infection rates after minimally invasive spinal surgery. J Neurosurg Spine 11: 471-476.

17. Postacchini $F$ (1999) Surgical management of lumbar spinal stenosis. Spine (Phila Pa 1976) 24: 1043-1047.

18. See $\mathrm{DH}$, Kraft GH (1975) Electromyography in paraspinal muscles following surgery for root compression. Arch Phys Med Rehabil 56: 80-83.

19. Sihvonen T, Herno A, Paljärvi L, Airaksinen O, Partanen J, et al. (1993) Local denervation atrophy of paraspinal muscles in postoperative failed back syndrome. Spine (Phila Pa 1976) 18: 575-581

20. Celik SE, Celik S, Göksu K, Kara A, Ince I (2010) Microdecompressive laminatomy with a 5-year follow-up period for severe lumbar spinal stenosis. J Spinal Disord Tech 23: 229-235.

21. Jensen MP, Turner JA, Romano JM, Fisher LD (1999) Comparative reliability and validity of chronic pain intensity measures. Pain 83: 157-162.

22. Fairbank JC, Couper J, Davies JB, O'Brien JP (1980) The Oswestry low back pain disability questionnaire. Physiotherapy 66: 271-273.

23. Englund J (2007) Lumbar spinal stenosis. Curr Sports Med Rep 6: 50-55. 